Molecules 2001, 6, M234

\title{
Bis[(3-methoxycarbonyl-5-methyl pyrazol)-1-yl Thiocarbonyl] Disulfide
}

\section{Abderrahman EI Idrissi, Karim Tebbji and Smaail Radi}

Laboratoire de chimie organique-Physique, Faculté des Sciences, Oujda, Morocco. Fax: +212 56500603. E-mail: Radi@sciences.univ-oujda.ac.ma

Received: 22 February 2001 / Accepted: 15 May 2001 / Published: 25 May 2001<smiles>COC(=O)c1cc(C)n(C(=S)SSC(=S)n2nc(C(C)=O)cc2C)n1</smiles>

This experiment is performed according to literature method [1-4]. 3(5)-Methoxycarbonyl-5(3)-pyrazole 1 (3.35 g, $0.024 \mathrm{~mol})$ and triethylamine $(6,65 \mathrm{~g}, 0.048 \mathrm{~mol})$ in ethanol solution were cooled to $5^{\circ} \mathrm{C}$ under stirring, carbon disulfide (3,65 g, $0.048 \mathrm{~mol})$ was added to the solution. After 1 hour of stirring, solid iodine $(2,8 \mathrm{~g}, 0.022 \mathrm{~mol})$ was added in portions and stirred until the colour disappeared completely. Then a methanolic solution of iodine was added dropwise until a faint colour persists. Excess of iodine was neutralized with $\mathrm{Na}_{2} \mathrm{~S}_{2} \mathrm{O}_{3}$ solution. The product was extracted with diethyl ether, washed thrice with water, dried over $\mathrm{Na}_{2} \mathrm{SO}_{4}$, filtered, and diethylether was evaporated at room temperature to give liquid compound $\mathbf{2}$.

Yield: (90\%).

${ }^{1} \mathrm{H}-\mathrm{NMR}\left(\mathrm{CDCl}_{3}\right) \mathrm{d}(\mathrm{ppm}): 2,30$ (s, 6H, $\left.\mathrm{CH}_{3}\right) ; 4,86$ (s, 6H, $\left.\mathrm{CO}_{2} \mathrm{CH}_{3}\right) ; 6,53$ (s, 2H, Hpy).

${ }^{13} \mathrm{C}-\mathrm{NMR}\left(\mathrm{CDCl}_{3}\right) \mathrm{d}(\mathrm{ppm}): 166(\mathrm{CO} 2), 126(\mathrm{C} 3), 52(\mathrm{O}-\mathrm{CH} 3)$

IR $\left(\mathrm{KBr}, \mathrm{cm}^{-1}\right)$ : $3200(-\mathrm{S}-\mathrm{S}-) ; 1720(\mathrm{C}=\mathrm{O}) ; 1240(\mathrm{C}=\mathrm{S}), 166\left(\mathrm{CO}_{2}\right), 126\left(\mathrm{C}_{3}\right), 52\left(\mathrm{O}_{-} \mathrm{CH}_{3}\right)$.

$\operatorname{MS}(\mathrm{m} / \mathrm{z}): 430[\mathrm{M}]^{+}$.

U.V.: $1_{\max }=290 \mathrm{~nm}(-\mathrm{C}=\mathrm{S})$.

\section{References}

1. Jones, R. G.; Hanret, M. J.; Lauglin, K. M. J. Org . Chem. 1954, 19, 1428.

2. Haque, S. A.; Clouet, G. Makromol. Chem. Phys. 1994, 195, 315-327.

3. Reiser, A. Photoreactive Polymer. The Science and Technology of Resist; Wiley: New York, 1986, p. 26.

4. El Idrissi, A.; Tebbji, K.; Radi, S. Molecules 2001, 6, M232.

Sample Availability: Available from the authors and from MDPI.

(C) 2001 MDPI. All rights reserved. Molecules website http://www.mdpi.org/molecules/ 